\title{
Pancreatic neuro-endocrine tumour
}

\author{
Wickramasuriya PKH ${ }^{1}$, Rajapakshe D C ${ }^{1}$, Lambiyas $\mathrm{P}^{1}$, Randunu LYP ${ }^{1}$
}

\section{Introduction}

Pancreatic neuro endocrine tumours are an interesting clinical entity which present challenges in both diagnosis and management, owing to its variable presentations. We present a $48 \mathrm{yr}$-old female who underwent surgical resection of a neuro endocrine tumour of the pancreas. The case report also describes the diagnostic challenges along with the management of this patient.

\section{Case report}

A 48-yr-old female presented to the surgical unit with intractable abdominal pain and backache. The backache started two months ago and was intermittent at the beginning. It was non radiating, dull pain which was worse at night. Abdominal pain followed the backache. It was a well-tolerated, dull pain initially. Pain acutely escalated to a severe, persistent, cramping like pain, which made her to seek medical attention. She also complained of recent onset weight loss and severe loss of appetite. She did not have any urinary symptoms, alteration of bowel habits, or fever.

She underwent resection of uterine fibroids 12 years back, and continues to have normal menstrual cycles. There was no clinical evidence of hypoglycemic episodes, or intractable dyspeptic symptoms to suggest a functioning pancreatic neuro-endocrine tumour. On examination abdomen was soft with mild guarding and tenderness in the epigastric, left hypochondriac and lumbar regions. No palpable masses were found.

White blood cell count, platelets, urine full report and culture, C-reactive protein, serum amylase, liver and renal function tests were normal. The blood sugar levels remained within normal limits. Ultra sound scan and computerised tomography (CT) of abdomen confirmed a $7.3 \times 7.9 \times 5.8 \mathrm{~cm}$ heterogeneous mass lesion at the tail of the pancreas, with multiple foci of calcification. Laterally the lesion extended to the splenic hilum and superiorly to the greater curvature of the stomach. The head, neck and proximal part of the body of the pancreas was normal. Four hypo dense lesions were seen in the segments iv-a and vii of the liver. Findings were suggestive of a malignant tumour of the pancreas, with possible multiple hepatic metastasis.
After an initial treatment with intravenous omeprazole, octreotide, metochlopromide and analgesics patient underwent distal pancreatectomy and successful total resection of the tumour. Histology confirmed a pancreatic neuro-endocrine tumour. Immunohistochemistry analysis is not available yet.

After an uneventful post-operative period, she was referred to an oncology centre for further management of liver metastasis.

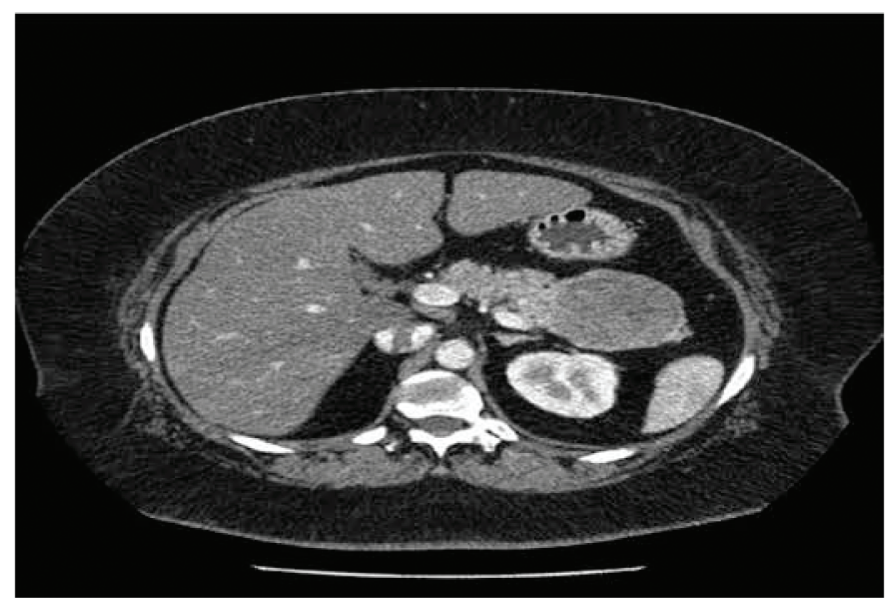

Figure $1 \mathrm{CT}$ Images showing the tumour with multiple hepatic metastases

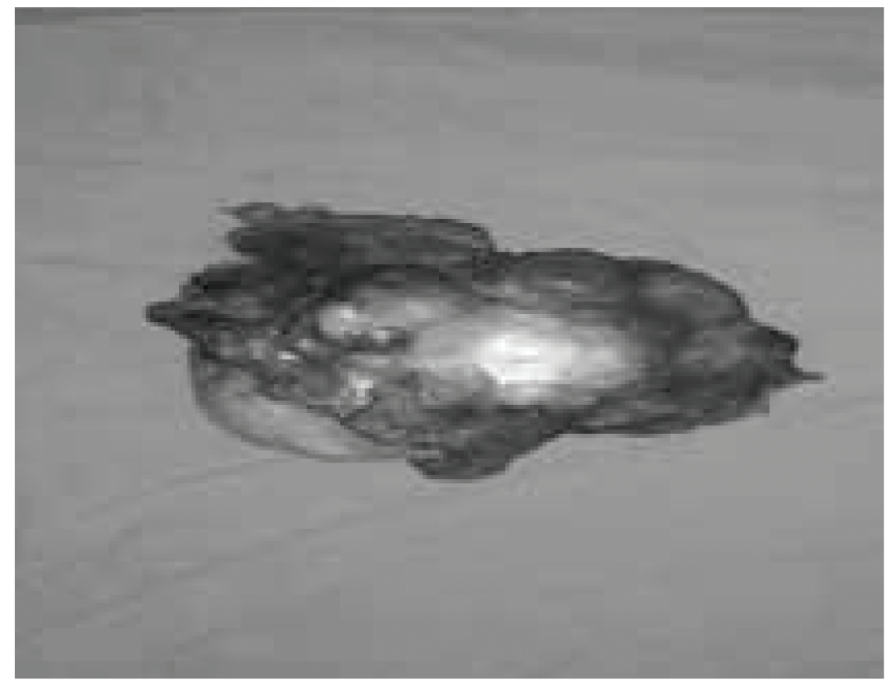

Figure 2 The dissected distal pancreas with the tumour

\section{General surgical unit District General Hospital Matara}

\section{https://orcid.org/0000-0002-1256-9427}

This is an open-access article distributed under the terms of the Creative Commons Attribution License, which permits unrestricted use, distribution and reproduction in any medium, provided the original author and source are credited. 


\section{Discussion}

Pancreatic neuro endocrine tumours account for nearly $5 \%$ of all clinically detectable pancraetic tumours ${ }^{1}$. A functioning pancreatic endocrine tumour will produce a specific clinical syndrome depending on the hormone it secrete, (an insulinoma causing hypoglyceamia, a gastrinoma causing Zollinger - Ellison syndrome) whereas a non functioning endocrine tumour(NF-PET) will cause non specific symptoms such as abdominal pain, obstructive jaundice and acute pancreatritis. These tumors also have a $10-20 \%$ association with multiple endocrine neoplasia type $1 .{ }^{1}$

Our patient did not show any clinical or biochemical evidence of a specific hormonal syndrome. Hence we managed her as a NF-PET. But since NF PETs can also express a certain amount of hormones, a conclusive diagnosis can only be made after immuno-histochemical analysis. The tumour size more than $5 \mathrm{~cm}$, presence of calcifications in the CT scan and a positive somatostatin receptor on scintigraphy suggest hormone activity. ${ }^{2}$

Diagnosis of NF-PETs could be challenging, because their non specific symptoms are often misinterpreted as benign conditions, and symptomatic liver metastasis could be the first presentation. Curative intent treatment for NF-PETs even in the presence of liver metastasis is aggressive surgical resection. ${ }^{3}$ Nearly $60 \%$ of cases can undergo curative resection ${ }^{4}$. The available surgical options include distal pancreatectomy, as in our patient, or pancreatico-duodenectomy with or without synchronous or metachronous resection of liver metastasis $^{4}$. An overall 5 year survival rate of $65 \%$ was noted in literature. ${ }^{5,6}$ However, the time of presentation and co morbid conditions of the patient may alter this outcome.

\section{Conclusion}

Pancreatic neuro endocrine tumours are a rare entity of intra abdominal malignancies which are often misinterpreted and mistreated. Diagnosis could be challenging especially in the absence of a specific clinical syndrome when the tumour is non-functioning. A high index of suspicion is needed for early diagnosis.

\section{References}

1. Bartsch, D.K., Fendrich, V., Langer, P., Celik, I., Kann, P.H. and Rothmund, M. (2005) Outcome of duodenopancreatic resections in patients with multiple endocrine neoplasia type 1. Annals of Surgery 242: 757-64.

2. Buetow PC, Parrino TV, Buck JL, et al. Islet cell tumors of the pancreas: pathologic-imaging correlation among size, necrosis and cysts, calcification, malignant behavior, and functional status. Am J Roentgenol. 1995;165:1175-9

3. Fendrich, V., Langer, P., Celik, I. et al. (2006) An aggressive surgical approach leads to long-term survival in patients with pancreatic endocrine tumors. Annals of Surgery 244: 845-51.

4. Fendrich V, Waldmann J, Bartsch DK, et al. Surgical management of pancreatic endocrine tumors. Nat Rev Clin Oncol. 2009;6:419-28.

5. Norton, J.A., Fraker, D.L., Alexander, H.R. et al. (2006) Surgery increases survival in patients with gastrinoma. Annals of Surgery 244: 410-19.

6. Dralle $H$, Krohn SL, Karges $W$, et al. Surgery of resectable nonfunctioning neuroendocrine pancreatic tumors. World J Surg. 2004;28:1248-60. 